\title{
Lenalidomide Induced Stroke in Multiple Myeloma
}

\section{KEYWORDS: stroke, lenalidomide, multiple myeloma, hypertension, hemiparesis}

Lenalidomide (Revlimid) is a novel agent used in treatment of multiple myeloma (MM) with an adverse effect of thromboembolic episodes, more commonly, deep vein thrombosis and pulmonary embolism [1]. Venous thromboembolism has emerged as one of the leading complications of the lifesaving drug [2]. In addition, there is an increased burden of lenalidomide induced stroke and myocardial infarction [3,4]. This is especially a concern as multiple myeloma is well recognized to be a prothrombotic state [4]. Recently, the antiangiogenic agents, in general, and lenalidomide in particular have been associated with stroke $[5,6]$. Furthermore, it was hypothesized that lenalidomide, a Thalidomide analog, induces less neurotoxicity compared to thalidomide $[7,8]$.

Tannemaat et al. reported an instance where a relatively young female suffered from stroke in one week after the administration of lenalidomide in the absence of other risk factors [9]. In the Chavez study, a patient died within 30 days of active treatment with lenalidomide due to an acute ischemic stroke [10]. In another instance, Scarpace et al. described one instance of transient ischemic attack (TIA) and two ischemic strokes treated with thalidomide 4 to 5 years after diagnosis of MM [11,12]. In addition, sudden death speculated due to stroke secondary to Lendalidomide has also been cited in literature [13]. This indicates that stroke; a dreadful complication of life changing drug can strike anytime (days, weeks, years) during treatment. There is an indication that intra-arterial light chain depositions may be responsible for the ischemic cerebrovascular events in these patients [9].

It can be seen both elderly and young adults, with increased frequency in newly diagnosed MM cases [2]. Clinical presentation can show considerable variation ranging from isolated headache to severe intracranial hypertension, hemiparesis, seizures, and encephalopathy. The severity of the complication may vary from reversible TIA to possible sudden death. An improvement of neurological symptoms is usually observed within 3-4 months after cessation of treatment with Thalidomide [14]. This can be compared with cisplatin which results in arterial ischemia and stroke which are more frequent within 10 days of treatment and following the first cycle of chemotherapy [15]. Similarly, intra-CSF or intravenous high-dose methotrexate, cyclophosphamide, 5-FU result in arterial ischemia. L-asparaginase on the other hand leads to hemorrhagic venous stroke mostly secondary to superior sagittal sinus or cortical vein thrombosis [16].

Adverse drug event reports received by US Food and Drug Administration (FDA) noted that lenalidomide for multiple myeloma was among the largest number of incomplete reports with the sole event term death in 2014 $(n=717)$ [15]. The stroke risk increases with immunomodulators like lenalidomide, further still with combination therapy of lenalidomide with corticosteroids and the maximum risk is associated with a combination therapy of lenalidomide and alkylating agents like doxorubicin [17]. An increased incidence of TEEs in 1 arm became apparent: $75 \%$ patients receiving dexamethasone and lenalidomide developed thromboembolic events (along with 1 ischemic stroke) after a median of 50 days, versus $0 \%$ on dexamethasone alone $(\mathrm{P}<0.001)[18,19]$. In the long-term follow-up of 704 patients with MM in 2 phase 3, randomized, clinical trials,
Shridhar $\mathrm{P}^{1 *}$, Khalil $\mathrm{R}^{2}$, Lasorda $D^{2}$, Chun $\mathrm{Y}^{1,3,4}$

${ }^{1}$ Department of Bioengineering, University of Pittsburgh, Pittsburgh, USA

${ }^{2}$ Department of Cardiology, Allegheny General Hospital, Pittsburgh, USA

${ }^{3}$ Department of Industrial Engineering, University of Pittsburgh, Pittsburgh, USA

${ }^{4} \mathrm{McGowan}$ Institute for Regenerative Medicine, Pittsburgh, USA

*Author for correspondence: Tel: 6502769952

pus8@pitt.edu 
the incidence of cerebrovascular events was $3.4 \%$, in patients treated with lenalidomide and dexamethasone compared with $1.7 \%$, in patients treated with dexamethasone alone [20]. A phase $1 / 2$ study of lenalidomide with vorinostat and dexamethasone, reported maximum tolerated dose was only $5 \mathrm{mg} /$ day due to patients experiencing grade 3 thrombocytopenia and grade 4 strokes [21].

It is recommended that all patients on lenalidomide and high dose (dexamethasone/ doxorubicin) should receive anticoagulation with either LMWH or full dose Warfarin with target INR of 2-3. According to recommendation from International Myeloma Working Group (IMWG), LMWH for all patients receiving lenalidomide and high-dose dexamethasone or doxorubicin and suggests that full-dose warfarin may be an alternative to LMWH. However, this was in the absence of clear data from randomized studies [9]. In a multi-center prospective, open label phase I/II trial, thromboembolic episodes including grade 4 stroke occurred despite LMWH administration to all patients [2224]. Due to the lack of prospective randomized clinical trials, different studies have used various anticoagulant prophylaxes, including fixed lowdose warfarin (1 mg or $1.25 \mathrm{mg}$ ), therapeutic doses of warfarin (international normalized ratio between 2.0 and 3.0), low molecular weight heparin, or low-dose aspirin [2]. As yet, no study has clearly demonstrated a significant superiority of one prophylactic regimen LMWH or full-dose warfarin effectively reduce the risk of VTEs.

In our experience, if the patient's INR was at target, we elected to maintain him on daily Plavix and monitor INR on regular basis. In patients who received prophylaxis with aspirin genetic variants of genes that are involved directly or indirectly in inflammatory response may be associated with increased risk of VTE [25]. Cohen et al. concluded that the benefits of aspirin are well documented for conditions like myocardial infarction and stroke [22]. It is well established that aspirin is effective in reducing the risk of myocardial infarction and stroke, but its role in reducing venous thrombosis is controversial [23]. A retrospective series of 83 patients who were treated with lenalidomide containing regimens showed asymptomatic venous thromboembolic event occurred in $18 \%$ despite aspirin prophylaxis. The authors further recommended serial monitoring of plasma D-dimer levels and early intervention may help to prevent symptomatic or lethal VTE events [26]. Moreover, ribavirin as a potential antithrombotic agent has also been suggested [27].

It is not uncommon for patients with plasma cell dyscrasias have a higher incidence of thromboembolic events associated with increased production of inflammatory cytokines such as CRP, IL-6 and TNF, procoagulant factors such as factor VIII and Von Willebrand factor, protein $\mathrm{C}$ resistance [28]. In addition, lenalidomide initiation in Myelodysplastic Syndrome with $5 \mathrm{q}$ deletion was found to be negatively associated with stroke [29]. It is very important that physicians should be aware lenalidomide carries a black box warning of significant stroke risks in patients with MM receiving lenalidomide and dexamethasone treatment [30]. Increased vigilance from clinicians for stroke or myocardial infarction is highly recommended in patients with multiple myeloma.

\section{REFERENCES}

Cruz MP. (2016) Lenalidomide (Revlimid): A Thalidomide Analogue in Combination with Dexamethasone for the Treatment of All Patients with Multiple Myeloma. Pharmacy and Therapeutics 41(2), 308.

Zamagni E, Brioli A, Tacchetti P, et al. (2011) Multiple myeloma, venous thromboembolism, and treatment-related risk of thrombosis. InSeminars in thrombosis and hemostasis 37(3), 209-219.

Singh A, Gajra A. (2011) Thromboembolism with immunomodulatory agents in patients with multiple myeloma. Cardiovascular \& Hematological Agents in Medical Chemistry 9(1), 7-13.

Lee GY, Lee YT, Yeh CM, et al. (2016) Risk of stroke in patients with newly diagnosed multiple myeloma: a retrospective cohort study. Hematological Oncology.

Ortín X, Rodríguez-Luaces M, Calabuig M, et al. (2006) Stroke in a multiple myeloma patient treated with thalidomide. Journal of Stroke and Cerebrovascular Diseases 15(6), 283-285.

Dearborn JL, Urrutia VC, Zeiler SR. (2014) Stroke and cancer-a complicated relationship. Journal of neurology \& translational neuroscience 2(1), 1039.

Grisold W, Cavaletti G, Windebank AJ. (2012) Peripheral neuropathies from chemotherapeutics and targeted agents: diagnosis, treatment, and prevention. NeuroOncology 14(4), 45-54.

Gay F, Hayman SR, Lacy MQ, et al. (2010)
Lenalidomide plus dexamethasone versus thalidomide plus dexamethasone in newly diagnosed multiple myeloma: a comparative analysis of 411 patients. Blood 115(7), 134350.

Tannemaat MR, Vries EP, Molendijk WJ, et al. (2011) Fatal ischemic stroke in a patient receiving Lenalidomide for multiple myeloma. Clinical neurology and neurosurgery 113(6), 488-9.

Chavez JC, Piris-Villaespesa M, Dalia S, et al. (2016) Results of a phase II study of Lenalidomide and rituximab for refractory/ relapsed chronic lymphocytic leukemia. Leukemia Research 47, 78-83.

Scarpace SL, Hahn T, Roy H, et al. (2005) Arterial thrombosis in four patients treated with thalidomide. Leuk Lymphoma. 46(2), 239-242. 
Libourel EJ, Sonneveld P, van der Holt B, et al. (2010). High incidence of arterial thrombosis in young patients treated for multiple myeloma: results of a prospective cohort study. Blood 116(1), 22-6.

Finsterer J, Höftberger R, Stöllberger C, et al. (2013) Sudden death possibly related to Lenalidomide given for cardiac and muscle AL amyloidosis secondary to light chain deposition disease. Journal of Oncology Pharmacy Practice 19(2), 170-4.

Barlogie B, Tricot G, Anaissie E, et al. (2006) Thalidomide and hematopoietic-cell transplantation for multiple myeloma. New England Journal of Medicine 354(10), 1021-30.

Moore TJ, Furberg CD, Mattison DR, et al. (2016) Completeness of serious adverse drug event reports received by the US Food and Drug Administration in 2014. Pharmacoepidemiology and drug safety 25(6), 713-8.

Taillibert S, Le Rhun E, Chamberlain MC. (2016) Chemotherapy-Related Neurotoxicity. Current neurology and neuroscience reports 16(9), 81.

Weiss L, Shah K, Yiu W, et al. (2009). Overview of Venous Thromboembolism (VTE) in Nearly 100,000 Patients Treated with Revlimid $^{\circledR}($ Lenalidomide). Blood. 114, 2881.

Zonder JA, Durie BG, McCoy J, et al. (2005) High
Incidence of Thrombotic Events Observed in Patients Receiving Lenalidomide (L)+ Dexamethasone (D)(LD) as First-Line Therapy for Multiple Myeloma (MM) without Aspirin (ASA) Prophylaxis. Blood 106, 3455.

Zonder JA, Barlogie B, Durie BG, et al. (2006) Thrombotic complications in patients with newly diagnosed multiple myeloma treated with Lenalidomide and dexamethasone: benefit of aspirin prophylaxis. Blood 108(1), 403-4.

Li W, Cornell RF, Lenihan D, et al. (2016) Cardiovascular complications of novel multiple myeloma treatments. Circulation. 133(9), 908-12.

Toumishey E, Prasad A, Dueck G, et al. (2015) Final report of a phase 2 clinical trial of Lenalidomide monotherapy for patients with T-cell lymphoma. Cancer. 121(5), 716-23.

Borgdorff P, Tangelder GJ. (2016) Arguments favoring low versus high dose aspirin in the prophylaxis of venous thromboembolism. Thrombosis research 139, 121-4.

Menon SP, Rajkumar SV, Lacy M, et al. (2008) Thromboembolic events with Lenalidomidebased therapy for multiple myeloma. Cancer 112(7), 1522-8.

Hopfinger G, Nösslinger T, Lang A, et al. (2014) Lenalidomide in combination with vorinostat and dexamethasone for the treatment of relapsed/refractory peripheral $\mathrm{T}$ cell lymphoma (PTCL): report of a phase I/II trial. Annals of hematology 93(3), 459-62.

Bagratuni T, Kastritis E, Politou M, et al. (2013) Clinical and genetic factors associated with venous thromboembolism in myeloma patients treated with Lenalidomide-based regimens. American journal of hematology 88(9), 765-70.

Isoda A, Sato N, Miyazawa Y, et al. (2015). Silent venous thromboembolism in multiple myeloma patients treated with Lenalidomide. International journal of hematology 102(3), 271-7.

Kamat AV (2014). Rivaroxaban Is an Effective and Well Tolerated Anti Thrombotic Agent in Patients on Lenalidomide Therapy and in Multiple Myeloma. Blood 124, 5095.

Palumbo A, Rajkumar SV, Dimopoulos MA, et al. (2008) Prevention of thalidomide-and Lenalidomide-associated thrombosis in myeloma. Leukemia 22(2), 414-23.

Zeidan AM, Gore SD, McNally DL, et al. (2013) Lenalidomide performance in the real world. Cancer 119(21), 3870-8.

Lenalidomide (Revlimid) (2016) Black box warning. 